\title{
From Art to Science: Advanced Cryocooling Technology for Biomolecular Cryocrystallography
}

\author{
Benjamin Apker ${ }^{a}$, Joyce Frank ${ }^{b}$, David Closs ${ }^{c}$, and Robert Thorne ${ }^{d}$ \\ ${ }^{a}$ MiTeGen, LLC, Ithaca, NY 14850, USA, benjamin.apker@mitegen.com \\ ${ }^{a}$ MiTeGen, LLC, Ithaca, NY 14850, USA, joyce@mitegen.com \\ ${ }^{\circ}$ MiTeGen, LLC, Ithaca, NY 14850, USA, david.c@mitegen.com \\ ${ }^{d}$ MiTeGen, LLC, Ithaca, NY 14850, USA, robert.thorne@mitegen.com
}

In biomolecular structure determination by X-ray crystallography, crystals are cooled to cryogenic temperatures, typically by manual plunge cooling in liquid nitrogen or by manual insertion in a cold nitrogen gas stream. Internal ice formation can destroy or severely degrade protein lattice diffraction. Ice forms rapidly in surface solvent, from moisture in ambient air that condenses on crystals during handling, and in the liquid nitrogen used for cooling and storage. Roughly $20 \%$ of PDB deposited data sets - and presumably a much larger fraction of all data sets - show structure factor errors consistent with ice contamination. Even when ice does not form, crystal mosaicities always worsen and diffraction resolution sometimes degrades.

Modest and highly variable cooling rates achieved in hand plunging can lead to substantial crystal-to-crystal nonisomorphism and substantial changes in the protein's conformation from its biologically relevant form. To prevent ice formation, crystals are grown or soaked in solutions containing cryoprotectants. Cryoprotectants can displace weakly bound ligands and complicate interpretation of weak density in electron density maps that may be associated with functionally relevant minor conformers, and reduce overall electron density contrast between solvent and protein. These problems are especially acute for crystals of membrane proteins and large complexes, which tend to have high solvent contents, large solvent cavities and more labile structures, and which often require larger cryoprotectant concentrations to prevent ice formation.

To address these problems, MiTeGen has developed NANUQ ${ }^{\mathrm{TM}}$, the world's most advanced instrument for crystal cryocooling. This instrument delivers the largest cooling rates possible using liquid nitrogen - roughly $50,000 \mathrm{~K} / \mathrm{s}$ for 50 micrometer crystals, or roughly 100 times larger than obtained in gas stream cooling - and also allows variable rate cooling at rates as low as $10 \mathrm{~K} / \mathrm{s}$. Ultrafast cooling reduces or eliminates the need for cryoprotectants, and more accurately captures the biologically relevant structure. Smaller cooling rates can facilitate internal solvent redistribution and biomolecular relaxations that can in some cases improve crystal order, especially when using large crystals. Cooling reproducibility is dramatically improved. Automated sample handling and storage eliminates crystal damage and loss and ice formation that occur during manual handling. Get the most out of your crystals, get everyone in your lab using the same reliable and reproducible protocols, and maximize the throughput of your crystallography pipeline using NANUQ's proven technology. 